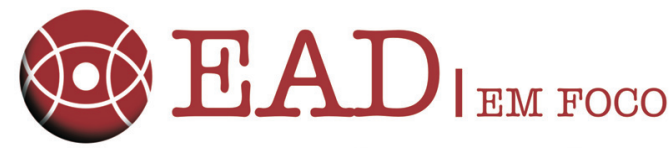

REVISTA CIENTÍFICA EM EDUCAÇÃO A DISTÂNCIA

\section{TUTORIA A DISTÂNCIA: CONSTRUINDO O CONHECIMENTO EM CURSOS ON-LINE}

Cristina Pfeiffer | pfeiffer@cederj.rj.gov.br

Professora Associada Doutora da Fundação CECIERJ, Professora Coordenadora da área Formação via Web da Diretoria de Extensão (CECIERJ), Doutora em Ciências pela COPPE/UFRJ, especialista em Atualização Pedagógica pelo CEP/UFRJ com estágio pós-doutoral em Tecnologias Educacionais na PUC-Rio.pfeiffer@cederj.rj.gov.br

Andreza Regina Lopes da Silva | andrezalopes.ead@gmail.com Mestranda do Programa de Pós-graduação em Engenharia e Gestão do Conhecimento da UFSC.

Sandra Menezes de Vasconcellos | vasconcellos321@oi.com.br

Professora da Rede Pública Estadual do RJ, tutora a distância na Área de Formação via web da Fundação CECIERJ, Especialista em Administração Escolar pelas Faculdades Integradas de Jacarepaguá.

Fernando José Spanhol | fernando.s@unitins.br/spanhol@led.ufsc.br

Vice reitor da Universidade Estadual do Tocantins, Professor Permanente do Programa de Pos-graduação em Engenharia e Gestao do Conhecimento da UFSC, Doutor em Midia e Conhecimento pela UFSC.

\section{Resumo}

Este estudo tem por objetivo apresentar as contribuições dos tutores a distância no processo de construção do conhecimento dos estudantes de cursos on-line, tendo por base a Espiral do Conhecimento proposta por Nonaka e Takeuchi (1997). Usando procedimentos metodológicos de uma pesquisa descritiva/bibliográfica, constatou-se que o processo de construção do conhecimento ocorre em espiral, iniciando em um nível mais individual e se ampliando para o nível coletivo, a partir das interações entre os tutores a distância e os estudantes. Os resultados obtidos também evidenciam a importância desses profissionais para o aumento da participação dos estudantes nas atividades assíncronas propostas no ambiente virtual de aprendizagem (AVA), bem como para a melhoria de seus índices de desempenho e aproveitamento. Os cursos analisados são oferecidos pela área de Formação Via Web da Diretoria de Extensão da Fundação Centro de Ciências e Educação Superior a Distância do Estado do Rio de Janeiro (CECIERJ).

\section{Palavras-chave}

Educação a distância. Conhecimento. Tutoria. Web 2.0. AVA. 


\section{TUTORING DISTANCE: \\ BUILDING KNOWLEDGE IN \\ COURSES ON-LINE}

\section{Abstract}

This study aims to present the contributions of distance tutors in the process of knowledge construction of students in on-line courses, based on the knowledge spiral proposed by Nonaka and Takeuchi (1997). The methodological procedures of a descriptive/literature research was used, and it shows that the process of knowledge construction occurs in a spiral, starting in a more individual level and expanding to the collective level, from the interactions among distance tutors and students. The results obtained also show the importance of these professionals to increase student participation in asynchronous activities proposed in the virtual learning environment (VLE), as well as in improving their performance and utilization rates. The analyzed courses are from the area of Web-Based Training offered by the Extension Courses' Board of the Fundação Centro de Ciências e Educação Superior a Distância do Estado do Rio de Janeiro (CECIERJ).

\section{Keywords:}

Distance Education. Knowledge.

Tutor. Web 2.0. VLE.

\section{Introdução}

As Tecnologias de Informação e Comunicação (TICs) se tornaram um elemento com presença constante na vida do ser humano, e seus adventos interferiram em grandes proporções: nas formas de agir, pensar e até mesmo na forma de relacionamento entre os indivíduos em um contexto social. Portanto, é impossível refletir sobre uma proposta educacional que busque formar pessoas críticas com capacidades e habilidades para atuar ativamente sem a integração de recursos tecnológicos em sua essência (FERREIRA, 2008).
Isso exige, no entanto, novas competências dos indivíduos, que devem estar preparados não só tecnologicamente, mas também em relação às formas de pensamento, ou melhor, competências cognitivas que os tornam ativos. A noção de competência designa uma capacidade de mobilizar diversos recursos significativos para enfrentar um tipo de situação (PERRENOUD, 2000).

Está na hora, portanto, de identificar o conjunto de competências e dos recursos das práticas profissionais e de escolher estrategicamente os que devem começar a ser construídos na formação inicial de profissionais reflexivos (PERRENOUD, 2002, p. 19). Assim:

\section{neste contexto, o conhecimento, matéria-prima da educação, torna-se um recurso estratégico para o desenvolvimento, e o mundo da educação não pode se isolar dos processos de transformação econômica e social, como também não pode deixar de incorporar, no sistema educacional, os novos re- cursos tecnológicos disponíveis (CASTRO, apud FERREIRA, 2005).}

Buscando formar professores para atender a essas demandas, novos ambientes de aprendizagem baseados nas TICs vêm sendo fonte de pesquisas e novos projetos nas universidades e escolas com objetivos cada vez mais amplos, a fim de possibilitar aos alunos o desenvolvimento dessas competências. Esse tipo de ambiente de aprendizagem tem sido cada vez mais reconhecido na área da Educação.

Berge (apud LITTO; FORMIGA, 2009, p. 73) aponta um modelo abrangente para a EAD baseado em quatro funções do professor on-line: pedagógica, social, gerencial e de suporte técnico. As quatro funções foram usadas como um modelo para discutir estratégias efetivas para a sala de aula virtual (PALLOFF; PRATT apud LITTO; FORMIGA, p. 73). No entanto, podemos afirmar que grande parte do êxito nessa metodologia de ensino se deve à ajuda mais característica, mais importante e mais decisiva aos estudantes: a Tutoria. Em um curso on-line, por exemplo, os tutores a distância são os principais atores de in- 
teração que, numa perspectiva ampla, abrangem uma ação de atores reflexivos capazes de criar, estimular, orientar, desenvolvendo a capacidade de aquisição e construção do conhecimento (OLIVEIRA; MILL; RIBEIRO, 2010).

A área de Formação via Web da Diretoria de Extensão da Fundação Centro de Ciências e Ensino Superior a Distância do Estado do Rio de Janeiro (CECIERJ), desde outubro de 2009, vem oferecendo a professores de escolas públicas e privadas do Estado do Rio de Janeiro cursos on-line gratuitos, em que, a partir da filosofia do "fazer-aprendendo", os professores aprendem a utilizar algumas aplicações gratuitas da web 2.0, que está se transformando em uma forma mais interativa de usar a web. Durante cada curso também é proposta uma reflexão e discussão sobre o uso dessas ferramentas como uma alternativa educacional para a prática docente desses professores.

O objetivo deste artigo é relatar o trabalho que vem sendo realizado pelos tutores a distância desses cursos de extensão on-line, mostrando suas contribuições no processo de construção do conhecimento dos professores cursistas. Usando como base a Espiral do Conhecimento, o presente artigo busca ampliar a discussão sobre a construção ${ }^{1}$ do conhecimento, que, segundo Nonaka $e$ Takeuchi (1997), é um processo em espiral, que começa a partir do nível individual e se amplia para diferentes seções, áreas e departamentos de uma organização, ou seja, sai do individual para o coletivo. $\mathrm{O}$ artigo está organizado da seguinte forma: inicialmente é feita uma introdução, seguida da fundamentação teórica, com os principais conceitos básicos; no item 3 se encontra a metodologia deste trabalho de pesquisa, seguida de uma aplicação com resultados apresentados no item 4; no item 5 são apresentadas algumas conclusões.

1 O termo construção do conhecimento é aqui trabalhado como sinônimo de criação, pois trabalha-se numa perspectiva de aprendizagem colaborativa que se dá com base na interação social.

\section{Fundamentação teórica}

Neste item é apresentada a fundamentação teórica na qual este artigo está baseado, em que se procura conceituar "criação do conhecimento" $e$ tutoria a distância.

\section{Construção do conhecimento}

Estamos hoje na sociedade do conhecimento, como já apontado por Drucker (1994), em que o recurso básico deixa de ser o capital, os recursos naturais ou mesmo a mão de obra, passando a ser o conhecimento, que, segundo Sveiby (1998), caracteriza-se por um conjunto de crenças verdadeiras e justificáveis.

Falar sobre conhecimento implica uma reflexão inicial sobre conhecimento tácito e explícito. Enquanto o primeiro é caracterizado como conhecimento pessoal e de difícil formalização, o segundo é estruturado podendo, mais facilmente, ser socializado. Por esse motivo, a criação, o compartilhamento, o armazenamento e a utilização do conhecimento são pontos amplamente discutidos e aplicados nas organizações nos dias de hoje (GROTTO, 2002).

Para Nonaka e Takeuchi (1997, p. 62), a construção do conhecimento consiste numa espiral que "surge quando a interação entre conhecimento tácito e conhecimento explícito eleva-se dinamicamente de um nível ontológico inferior até níveis mais altos". De acordo com os autores, essa espiral está relacionada a quatro quadrantes de conversão do conhecimento, como apresentado na figura 1 .

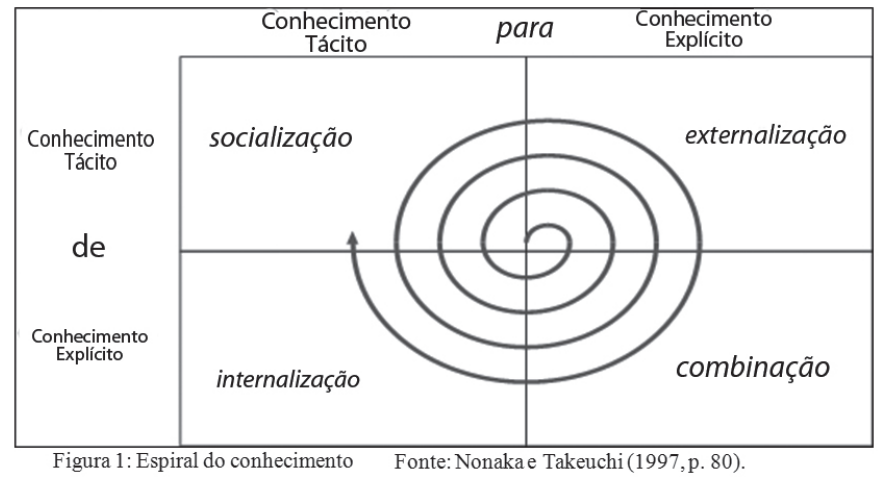


A espiral pode iniciar-se em qualquer um dos quatro quadrantes, desde que haja interação entre o conhecimento tácito e o conhecimento explícito. Comumente o início da construção do conhecimento com base na espiral proposta por Nonaka e Takeuchi (1997) ocorre na socialização, em que se caracteriza um cenário de interação com o objetivo de compartilhar o conhecimento tácito, ou seja, o processo de troca de experiência. $\mathrm{Na}$ externalização ocorre o compartilhamento do conhecimento através de diálogo ou da reflexão coletiva, em que os membros da equipe podem explicitar o conhecimento de forma não direta. Já o terceiro quadrante, a combinação, caracteriza-se pelo momento da disponibilização do conhecimento recém-criado juntamente com o já existente proveniente de outras seções da organização em uma rede. E, por fim, a internalização implica o conhecimento do indivíduo por meio do aprender fazendo (NONAKA; TAKEUCHI, 1997).

Bhatt (2002) acrescenta ainda que nesse processo interativo o indivíduo, além de ampliar o seu conhecimento, também contribui para a ampliação do conhecimento do grupo, ou seja, o conhecimento que é internalizado num grupo, numa comunidade, não é fruto de um indivíduo apenas, mas é resultado da interação entre eles.

Por fim, identifica-se que por meio desse processo cíclico evidenciado nos quatro quadrantes da espiral do conhecimento obtém-se o processo de construção do conhecimento, que, num cenário macro da EAD, é alavancado pelo uso de técnicas, métodos, mídias e atores envolvidos em uma determinada área de atuação de conhecimento.

Sendo assim, deve-se destacar a importância da mediação na tutoria a distância durante o processo de construção do conhecimento baseado na troca de ideias e experiências fundamentadas no diálogo, na participação coletiva.

Como afirma Lins (2005), o tutor é o mediador, o provocador de uma aprendizagem significativa, levando o estudante a interações que sejam capazes de desenvolvê-lo, tornando-o um indivíduo cada vez mais consciente e autônomo em seus di- reitos e deveres.

\section{Tutoria a distância}

A Lei de Diretrizes e Bases da Educação (LDB), no art. 47, § $3^{\circ}$ (apud CAMPOS, 2008), define EAD como uma atividade pedagógica caracterizada por um processo de ensino-aprendizagem realizado com mediação docente e a utilização de recursos didáticos sistematicamente organizados, apresentados em diferentes suportes tecnológicos de informação e comunicação, os quais podem ser utilizados de forma isolada ou combinadamente, sem a frequência obrigatória de alunos e professores.

Portanto, muitos atores participam de um sistema de EAD, que, segundo Litto (2010), devem fazer parte de uma equipe multidisciplinar constituída por: gerente, especialista em conteúdo (professor-autor), especialista em mídia, designer gráfico, bibliotecário e programador para ambiente virtual de aprendizagem (AVA), além de um sistema de tutoria.

Num sistema de EAD on-line os tutores a distância são os principais atores de interação, que, numa perspectiva ampla, abrangem uma ação de atores reflexivos capazes de criar, estimular $e$ orientar, desenvolvendo a capacidade de aquisição e construção do conhecimento (OLIVEIRA; MILL; RIBEIRO, 2010). Brasil (2007) aponta como condição essencial para que o tutor exerça sua função o domínio do conteúdo aliado a dinamismo, visão crítica e global, além da capacidade para estimular a busca por novos conhecimentos $e$ habilidades. E isso exige formação continuada e autonomia, que são características importantes para a excelência de suas atividades (OLIVEIRA; MILL; RIBEIRO, 2010). Silva et al. (2010) relaciona a figura do tutor ao ensino mais individualizado, contribuindo para que os alunos tenham uma aprendizagem significativa e contextualizada.

Sendo assim, existem algumas características essenciais para a realização das atividades de tutoria (ARETIO, 2002). São elas: 1) acompanhamento do desempenho do estudante durante as 
atividades; 2) auxílio e orientação aos estudantes buscando sempre esclarecer todas as dúvidas. Por exemplo, em um AVA esse profissional esclarece as dúvidas dos estudantes por meio das diferentes ferramentas de EAD (síncronas ou assíncronas), de acordo com o projeto pedagógico (BRASIL, 2007); 3) estímulo à busca por informações essenciais e complementares; 4) busca pela motivação do estudante; 5) domínio dos conteúdos didáticos oferecidos no curso.

\section{Métodos de pesquisa e técnicas de análise}

A metodologia da pesquisa adotou o delineamento proposto por Vergara (2003):

1) quanto aos fins, a pesquisa tem natureza exploratória e descritiva; $e$

2) quanto aos meios, trata-se de uma pesquisa bibliográfica.

Para Triviños (1992) a abordagem qualitativa caracteriza-se por atividades de investigação específicas. Chizzotti (2001) aponta ainda que, nesta metodologia, o pesquisador é um ativo participante na pesquisa, compreensão e interpretação dos dados.

Netto (2006) afirma que a finalidade da pesquisa descritiva é observar, registrar e analisar sem haver a necessidade de entrar no mérito dos conteúdos.

Neste artigo utiliza-se a pesquisa descritiva para retratar a prática da tutoria a distância dos cursos de extensão on-line da área de Formação Via Web e sua contribuição na construção do conhecimento dos professores cursistas. A coleta de dados deu-se por meio da observação da coordenação da área, coordenação de tutoria e dos tutores a distância.

Quanto aos meios, trata-se de uma pesquisa bibliográfica, por basear-se em um estudo sistematizado, desenvolvido com base em material publicado e, que segundo Jung (2004, p. 160), objetiva "conhecer as diversas formas de contribuições científicas existentes que foram realizadas sobre determinado assunto ou fenômeno".

Tutoria a distância na área de Formação Via Web
De setembro a dezembro de 2009, a Diretoria de Extensão da Fundação CECIERJ ofereceu um curso on-line intitulado Formação Continuada em Tecnologias Educacionais via Web (FCTEWeb). A proposta do curso era usar a filosofia do "fazer-aprendendo" com os professores cursistas para capacitá-los no uso de algumas ferramentas web 2.0 (Google Docs, Twitter e blog). Diante da boa aceitação, esse curso foi oferecido novamente em 2010, sendo configurado em dois módulos; no módulo 1 trabalhou-se Google Docs e Twitter; no módulo 2, trabalhou-se com blog. Isso aconteceu em função das sugestões feitas numa pesquisa de satisfação realizada com os participantes ao final do curso em 2009.

Para atender à demanda de professores que estavam em busca de um aperfeiçoamento em modelos de design instrucional e em mediação de ambientes virtuais de aprendizagem, a Diretoria de Extensão da Fundação CECIERJ proporcionou a criação da área Formação via Web com o oferecimento dos dois módulos do curso FCTEWeb e de mais cursos on-line: Mediando cursos em ambientes virtuais de aprendizagem e Modelos de DI para material didático: potencializando o processo de ensino-aprendizagem em EAD. Vale ressaltar que a metodologia utilizada nos cursos novos seguiu a mesma filosofia do "fazer-aprendendo". Os objetivos da área Formação via Web são:

- Refletir e discutir sobre uma forma mais interativa de usar a web 2.0 como uma alternativa educacional para a prática docente - Curso FCTEWeb módulos 1 e 2;

- Preparar professores para a elaboração de projetos educacionais baseados em modelos de design instrucional para o aprimoramento da prática docente - Cursos Modelo de DI para material didático: potencializando o processo de ensino-aprendizagem em EAD;

- Preparar professores para a mediação de cursos em ambientes virtuais de aprendizagem (AVAs), apresentando os principais recursos do AVA Moodle que podem ser incorporados a um curso on-line - Curso Mediando cursos em am- 
bientes virtuais de aprendizagem.

Os cursos são gratuitos, com carga horária de 30 horas, numa proposta semestral e destinados a profissionais de instituições públicas e privadas que atuam na área da Educação.

Para tanto, a área conta com uma coordenadora geral, uma coordenadora de tutoria, um administrador do ambiente Moodle e cinco tutores a distância, além de outros profissionais que participaram da etapa de planejamento e elaboração dos cursos, tais como: designer gráfico, web designer e revisor textual, formando, dessa forma, uma equipe multidisciplinar.

A tutoria, que ocorre em um regime totalmente a distância, é formada por cinco tutores que acompanham turmas com 70 alunos em média. A formação profissional desses tutores inclui capacitação em nível de graduação e pós-graduação (especialização, mestrado) em diferentes áreas do conhecimento compatíveis com a proposta dos cursos. A função de cada tutor a distância da área Formação via Web, seguindo Oliveira et al (2010), é ser um ator reflexivo capaz de criar, estimular, orientar, desenvolvendo a capacidade de aquisição e construção do conhecimento dos cursistas e trabalhando, essencialmente, as ferramentas designadas em cada um dos cursos para auxiliar, facilitar e ampliar a atuação do cursista em sua prática docente.

As contribuições dos tutores a distância da área de Formação via Web no processo de construção do conhecimento dos professores cursistas foram identificadas com base na observação participante, nas ações de prática da tutoria aos quatro quadrantes da espiral do conhecimento apresentadas e descritas anteriormente, segundo Nonaka e Takeuchi (1997), conforme descrito a seguir:

- Socialização: a primeira semana dos cursos é destinada à leitura de um texto de abertura ao tema do curso e à participação num fórum de integração entre os participantes e os tutores, auxiliando a avaliação diagnóstica e caracterização da turma. Os cursistas, estimulados pelo tutor, devem responder no fórum a quatro perguntas:
1) Quem sou? (breve descrição com características pessoais e profissionais); 2) Quais minhas expectativas em relação a este curso?; 3) Como pretendo administrar meu tempo para a realização deste curso?; e 4) O que me chamou mais a atenção após ler o texto de abertura? Esse período, segundo Nonaka e Takeuchi (1997) pode ser caracterizado como um cenário de interação com o objetivo de compartilhar o conhecimento tácito. O mesmo processo ocorre entre os tutores $e$ a as coordenações (geral e tutoria) do curso, em um fórum da coordenação que tem por objetivo compartilhar dúvidas e problemas a serem solucionados durante o curso, além de conquistas e trocas informais. Essas ações podem ser identificadas como sendo o primeiro quadrante da espiral do conhecimento (figura 1), uma vez que nesse momento existe a troca de experiências vividas tanto entre os estudantes e tutores quanto entre a própria equipe, por meio do compartilhamento do seu conhecimento tácito. A experiência é significativa em termos da quantidade de mensagens postadas nos fóruns de integração dos quatro cursos. Por exemplo, no primeiro semestre de 2011 o fórum de integração contou com um total de 1.705 mensagens postadas (divididas em 3 grupos) no curso FCTEWeb - módulo 1; contou com 297 mensagens postadas (dividas em 2 grupos) no curso FCTEWeb - módulo 2; teve 276 mensagens postadas no curso Modelos de DI para material didático: Potencializando o processo de ensino-aprendizagem em EAD. Totalizou-se nesse fórum 562 mensagens, e no curso Mediando cursos em AVA contou-se com 570 mensagens postadas.

- Externalização: a partir da segunda semana, nos quatro cursos da área, os tutores procuram estimular uma maior interação entre os estudantes. Usando um texto-base e textos de apoio com uma conceituação do tema, o objetivo desta etapa é a construção de novos conhecimentos conceituais pelos cursistas, com base na explicitação dos conhecimentos tácitos até então existentes. Para alcançar tal objetivo, trabalha-se de forma colaborativa, propondo questões instigantes 
em um fórum e levando o aluno a compartilhar a sua experiência, ou seja, o cursista compartilha seu conhecimento tácito com todo o grupo. Nesse momento o tutor deve acessar diariamente o curso, procurando responder, em linhas gerais, a todas as colocações dos cursistas, bem como explicitar sua prática, além de propor novas questões a partir do exposto pelo grupo para dinamizar o fórum. Todo esse compartilhamento é instigado pelos tutores, que trabalham constantemente de forma a estimular a participação.

- Combinação: como a metodologia dos cursos trabalha com base no aprender-fazendo, esta etapa trabalha com o conhecimento já explicitado pelo material didático disponibilizado pelos conteudistas e principalmente com o conhecimento construído pelos cursistas com a troca de saberes entre tutor e cursistas. Esses momentos foram sendo oportunizados e estimulados pelas práticas de construção colaborativas por meio de ferramentas como: Google Docs, Twitter e blog, além do fórum de discussão. Aqui todos os atores envolvidos se enriquecem com o processo de combinação do conhecimento, que deixou de ser individual e passou a ser coletivo.

- Internalização: este momento acontece na reta final do curso e é caracterizado pela vivência prática dos resultados até então alcançados pelo grupo associados ao processo de aprendizagem, também denominado conhecimento operacional. Isso fica evidenciado nos relatos finais do estudante, que explicita o conhecimento adquirido $e$ o seu saber construído ao longo do curso. Nessa reta final dos cursos trabalha-se também na proposta colaborativa, só que agora conta-se com uma maior contribuição dos cursistas, o que pode ser observado em alguns depoimentos obtidos no processo de avaliação e autoavaliação, que evidenciam que o conhecimento explicitado se tornou implícito, conforme mostrado no Quadro 1:

\section{Quadro 1: Relatos de cursistas dos cursos da área Formação via Web (CECIERJ)}

\begin{tabular}{|c|c|}
\hline Participante $\mathrm{X}$ & $\begin{array}{l}\text { Utilizei a wiki. Achei que eles ficaram um pouco enrolados no } \\
\text { início. Quando iam escrever as suas partes, apagavam as } \\
\text { anteriores e até eles entrarem no ritmo, levou-se um tempo. } \\
\text { Mas no fim, todos gostaram da experiência e cumpriram a } \\
\text { tarefa de forma satisfatória. }\end{array}$ \\
\hline Participante $\mathrm{Z}$ & $\begin{array}{l}\text { A interação constante } e_{\text {m }} \text { as mensagens muito enriquecedoras } \\
\text { contribuíram para o objetivo do fórum e a construção do } \\
\text { conhecimento do grupo. }\end{array}$ \\
\hline Participante $Y$ & $\begin{array}{l}\text { O curso fortaleceu meu interesse em ampliar meu } \\
\text { conhecimento e já estou fazendo uma pós em Tecnologias e } \\
\text { EAD. Também influenciou em minha prática pedagógica. }\end{array}$ \\
\hline
\end{tabular}

Fonte: Dados primários.

Com base na experiência dos cursos de extensão on-line da área Formação Via Web, observa-se que há indícios da convergência entre os quatro quadrantes apresentados por Nonaka e Takeuchi (1997) e que o conhecimento cresce exponencialmente à medida que ele é socializado, externalizado, combinado para então ser internalizado, desdobrando assim o processo de aprendizagem, que envolve tanto pessoas e processos quanto tecnologia, estando alinhados com o objetivo de cada curso. Por exemplo, o momento de socialização contou com um índice considerado satisfatório, dado o número expressivo de estudantes que foram para a fase seguinte dos cursos que consistiam na externalização do conhecimento, até então considerado tácito. Ao trabalharem de 
forma colaborativa por meio dos recursos tecnológicos da web 2.0, pôde-se explicitar e compartilhar o conhecimento, contribuindo, por fim, para a aprendizagem, quando houve a internalização.

\section{Conclusão}

Com base nas constatações empíricas e teóricas apresentadas no presente estudo, ficou evidenciado que o tutor a distância exerce papel significativo e influente num projeto de $\mathrm{EAD}$, visto que seu trabalho vai além de uma atuação técnica, abrangendo, assim, os quatro quadrantes da criação do conhecimento propostos por Nonaka e Takeuchi (1997): socialização, externalização, combinação e internalização do conhecimento.

Os tutores a distância dos cursos de extensão on-line da área Formação via Web oferecido pela Diretoria dos Cursos de Extensão da Fundação
CECIERJ desempenham um importante papel, pelo estímulo e o diálogo entre as partes, procurando manter o interesse permanente dos alunos para a construção coletiva do conhecimento. Foi evidenciado também que os tutores a distância são um facilitador desse processo, mediante o estimulo e o diálogo entre as partes, procurando manter o interesse permanente dos alunos para a construção coletiva do conhecimento.

Sabe-se que o presente artigo não pode ser generalizado por se tratar de um estudo de caso, específico. Portanto, como trabalho futuro, sugere-se a observação de outros grupos de tutores a distância, buscando evidências de que a atuação desses atores possa potencializar a construção, o uso e a disseminação do conhecimento, com base na combinação dos quatro quadrantes da espiral do conhecimento.

\section{Referências:}

ARETIO, Lorenzo Garcia. Educación a distancia hoy. Madrid: UNED, 1994.

La Educación a Distancia: de La teoría a La práctica. Barcelona: Ariel, 2002. AZEVEDO, Wilson. Panorama Atual da Educação a Distância no Brasil. Conect@ - Revista on-line de Educação a Distância. Disponível em: <http://www.revistaconecta.com/conectados/wilson_seminario.htm>. Acesso em: 18 jan. 2011.

BRASIL. Ministério da Educação. Referenciais de qualidade para educação superior a distância. Secretaria de Educação a Distância, Brasília: MEC, 2007.

CAMPOS, Fernanda C. A.; COSTA, Rosa M. E. M. ; SANTOS, Neide. Fundamentos da Educação a Distância, mídias e ambientes virtuais. Juiz de Fora: Editar. 2007.

CHIZZOTTI, Antonio. Pesquisa em ciências humanas e sociais. São Paulo: Cortez, 2001. DRUCKER, P. F. Sociedade pós-capitalista. São Paulo: 1994.

FERREIRA, Alan Silva. Curso de capacitação na modalidade de ensino a distância: a construção do conhecimento no ensino de geografia apoiado pelas tecnologias da informação e comunicação. Trabalho de Conclusão do Curso de Pós-Graduação lato sensu em Especialização em Educação a Distância. SENAC/RJ. 2008.

A contribuição da robótica para o desenvolvimento das competências cognitivas superiores: no contexto dos projetos de trabalho. Monografia de Conclusão de Curso em Licenciatura em Pedagogia. Universidade Estácio de Sá. Rio de Janeiro. 2005.

GROTTO, Daniela. O compartilhamento do conhecimento nas organizações. In: ANGELONI, Maria Terezinha. Organizações do conhecimento: infraestrutura, pessoas e tecnologias. São Paulo: Saraiva, 2002.

LAKATOS, Eva Maria; MARCONI, Marina de Andrade. Metodologia cientifica: ciência e co- 
nhecimento cientifico, métodos científicos, teoria, hipóteses e variáveis. $2^{a}$ ed. São Paulo: Atlas, 1992. LINS, Maria Judith Sucupira da Costa. A aprendizagem e a tutoria. São Paulo: SENAC Nacional, 2005.

LITTO, F. Aprendizagem a distância. São Paulo: Imprensa Oficial, 2010.

LITTO, Frederic M.; FORMIGA, Marcos (organizadores). Educação a Distância: o estado da arte. São Paulo: Pearson Prentice Hall. 2009.

MACEDO, Marcelo et al. Gestão do conhecimento organizacional. Florianópolis: 2010.

MOORE, Michael. KEARSLEY, Greg. Educação a distância: uma visão integrada. São Paulo: Cengage Learning, 2008.

NETTO, Alvim Antônio de Oliveira. Metodologia da pesquisa científica: guia prático para apresentação de trabalhos acadêmicos. Florianópolis: Visual Books, 2006.

NONAKA, I.; TAKEUCHI, H. A criação de conhecimento na empresa. Rio de Janeiro: Campus, 1997.

OLIVEIRA, Marcia R. G.; MILL, Daniel; RIBEIRO, Luis Roberto de C. A tutoria como formação docente na modalidade de Educação a Distância. In: MILL, Daniel;

RIBEIRO, Luis Roberto de C.; OLIVEIRA, Marcia R. G. Polidocência na Educação a Distância: múltiplos enfoques. São Paulo: EdUFSCar, 2010.

REIS, Ana Maria Viegas. Ensino a distância: megatendência atual abolindo preconceitos. São Paulo: Imobiliária, 1996.

SVEIBY, Karl Erick. A nova riqueza das organizações: gerenciando e avaliando patrimônios de conhecimento. Rio de Janeiro: Campus, 1998.

SILVA, Andreza R. Lopes da et al. A terminologia da EAD: conceito e compreensão. Anais do Congresso internacional de Educação a Distância. Foz do Iguaçu: ABED, 2010a, 16, CD.

TRIVIÑOS, Augusto N. S. Introdução à pesquisa em Ciências Sociais: a pesquisa qualitativa em Educação. São Paulo: Atlas, 1992.

VYGOTSKY, Lev S. A formação social da mente. São Paulo: Martins Fontes, 1987. 